Research Article

\title{
Computing Eccentricity-Based Topological Indices of 2-Power Interconnection Networks
}

\author{
Muhammad Imran (D), ${ }^{1}$ Muhammad Azhar Iqbal, ${ }^{2}$ Yun Liu, ${ }^{3}$ Abdul Qudair Baig, \\ Waqas Khalid, ${ }^{5}$ and Muhammad Asad Zaighum ${ }^{2}$ \\ ${ }^{1}$ Department of Mathematical Sciences, College of Science, United Arab Emirates University, P. O. Box 15551, Al Ain, UAE \\ ${ }^{2}$ Department of Mathematics and Statistics, Riphah International University, Islamabad, Pakistan \\ ${ }^{3}$ School of Electronic Engineering, Huainan Normal University, Huainan 232038, China \\ ${ }^{4}$ Department of Mathematics and Statistics, Institute of Southern Punjab, Multan, Pakistan \\ ${ }^{5}$ Punjab College of Science and Commerce, Attock Campus, Pakistan
}

Correspondence should be addressed to Muhammad Imran; imrandhab@gmail.com

Received 11 October 2019; Accepted 11 May 2020; Published 13 June 2020

Academic Editor: Juan L. G. Guirao

Copyright ( $\odot 2020$ Muhammad Imran et al. This is an open access article distributed under the Creative Commons Attribution License, which permits unrestricted use, distribution, and reproduction in any medium, provided the original work is properly cited.

\begin{abstract}
In a connected graph $G$ with a vertex $v$, the eccentricity $\varepsilon(v)$ of $v$ is the distance between $v$ and a vertex farthest from $v$ in the graph $G$. Among eccentricity-based topological indices, the eccentric connectivity index, the total eccentricity index, and the Zagreb index are of vital importance. The eccentric connectivity index of $G$ is defined by $\xi(G)=\sum_{v \in V(G)} d(v) \mathcal{E}(v)$, where $d(v)$ is the degree of the vertex $v$ and $\varepsilon(v)$ is the eccentricity of $v$ in $G$. The topological structure of an interconnected network can be modeled by using graph explanation as a tool. This fact has been universally accepted and used by computer scientists and engineers. More than that, practically, it has been shown that graph theory is a very powerful tool for designing and analyzing the topological structure of interconnection networks. The topological properties of the interconnection network have been computed by Hayat and Imran (2014), Haynes et al. (2002), and Imran et al. (2015). In this paper, we compute the close results for eccentricity-based topological indices such as the eccentric connectivity index, the total eccentricity index, and the first, second, and third Zagreb eccentricity index of a hypertree, sibling tree, and $X$-tree for $k$-level by using the edge partition method.
\end{abstract}

\section{Introduction}

The advancement of large-scale integrated circuit technology has enabled the construction of complex interconnection networks. Graph theory provides a fundamental tool for designing and analyzing such networks. Graph theory and interconnection networks provide a thorough understanding of these interrelated topics [1-3] The architecture of an interconnected network is represented by a graph, where nodes represent the processors and edges represent the links between processors. Electric power companies need to continually monitor the state of their systems as in the case of the voltage magnitude at loads and the machine phase angle at generators. In the electric power system, a vertex represents an electric node and an edge represents a transmission line joining two electrical nodes $[4,5]$. Chemical graph theory is a branch of mathematical chemistry in which we apply tools of graph theory to model the chemical phenomenon mathematically. This theory contributes a prominent role in the fields of chemical sciences. A molecular or chemical graph is a simple finite graph in which vertices denote the atoms and edges denote the chemical bonds in the underlying chemical structure. A topological index is actually a numeric quantity associated with chemical constitution purporting for correlation of the chemical structure with many physiochemical properties, chemical reactivity, and biological activities. Let $G$ be a graph with $u$ and $v$ being the vertices of $G$, then the distance $d(u, v)$ is 
defined as the shortest length between $u$ and $v$. The eccentricity $\varepsilon(v)$ of a vertex $v \in V(G)$ is defined as

$$
\varepsilon(v)=\max \{d(v, w) \mid w \in V(G)\} .
$$

The minimum eccentricity in a graph $G$ is known as the radius $G \operatorname{rad}(G)$, while the maximum eccentricity in a graph $G$ is known as the diameter $2 p-2$. Topological descriptors play an important role in the quantitative structure-activity (QSAR) and structure-property (QSPR) study. Topological indices, which are based on eccentricity of the vertices in a graph $G$, are known as eccentricity-based topological indices. The eccentric connectivity index [6] of $G$ is defined by

$$
\xi(G)=\sum_{v \in V(G)} d(v) \varepsilon(u)
$$

where $d(v)$ is the degree of the vertex $v$ and $\varepsilon(v)$ is the eccentricity of $v$ in $G$.

The total eccentricity index [7] is defined as the summation of eccentricity of each vertex in graph $G$. In other words, when the vertex degrees are not considered in the eccentric connectivity index, then we obtain the total eccentricity index:

$$
\zeta(G)=\sum_{u \in V(G)} \varepsilon(u)
$$

where $\varepsilon(v)$ is the eccentricity of $u$ in $G$.

Zagreb indices [8] have been introduced more than thirty years ago. Let $G$ be a graph with $u$ and $v$ being the vertices of $G$; then the Zagreb indices are defined as follows:

$$
\begin{aligned}
& M_{1}(G)=\sum_{v \in V(G)}(d(v))^{2}, \\
& M_{2}(G)=\sum_{u v \in E(G)}(d(u) d(v)) .
\end{aligned}
$$

Some new modified versions of Zagreb indices [9] are expressed in terms of eccentricity as follows:

$$
\begin{aligned}
M_{1}^{\star}(G) & =\sum_{u v \in E(G)}(\varepsilon(u)+\varepsilon(v)), \\
M_{1}^{\star \star}(G) & =\sum_{v \in V(G)}(\varepsilon(v))^{2}, \\
M_{2}^{\star}(G) & =\sum_{u v \in E(G)}(\varepsilon(u) \varepsilon(v)) .
\end{aligned}
$$

On further results for certain degree-based topological indices of networks and nanostructures, consult [1, 10-13]. A tree is a connected acyclic graph, and the common type of a tree is a binary tree. A binary tree is made of nodes, where each node contains a left reference, a right reference, and a data element. The top most node is called the root. The vertex of the binary tree has three fields. The first field represents data, while the second and third contain information of the left and right sons of the vertex. If each internal vertex/node has exactly two descendents, then the binary tree is said to be a complete binary tree as shown in Figure 1. The basic skeleton of the hypertreek-level is a complete binary tree $\mathrm{HT}(k)$. The nodes of the trees are labeled in such a way that root node has label 1 . The root is at level 0 . Labels

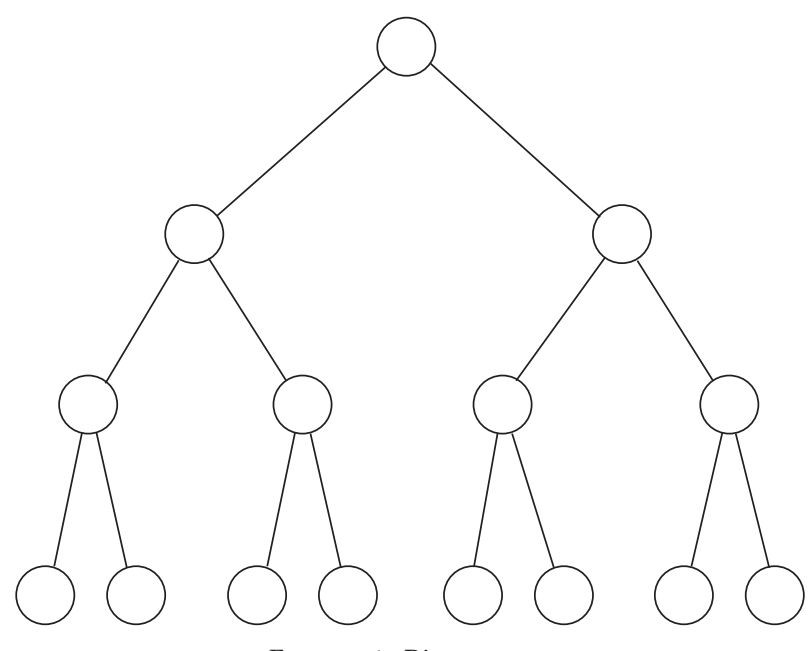

Figure 1: Binary tree.

of the left and right children are formed by appending 0 and 1 , respectively, to the labels of the parent node. Here, the children of the nodes $x$ are labeled as $2 x$ and $2 x+1$. Additional links in a hypertree are horizontal, and two nodes in the same level of the tree are joined if their label difference is $2^{i-2}$ [14]. The hypertree $k$-level HT $(k)$ shown in Figure 2 has vertices $2^{k+1}-1$ and edges $3\left(2^{k}-1\right)$.

\section{Main Results}

In this section, we compute the close results for eccentricitybased topological indices such as the eccentric connectivity index, the total eccentricity index, and the first, second, and third Zagreb eccentricity index of a hypertree, sibling tree, and $X$-tree for $k$-level by using the edge partition method. The molecular topological descriptors of fullerenes and several interconnection networks have been already computed in the literature $[1-3,15]$.

In the next theorem, an exact expression for the eccentric connectivity index for a binary tree is computed.

Theorem 1. Consider the graph $G \cong H T(k)$, then the eccentric connectivity index is equal to

$$
\xi(G)=2 k+4 \sum_{i=1}^{k-1} \sum_{p=k}^{2 k-2} 2^{i} p+2^{k+1}(2 k-1)
$$

Proof. In order to prove the above result, we use the formula of the eccentric connectivity index:

$$
\xi(G)=\sum_{v \in V(G)} d(v) \mathcal{\varepsilon}(v) .
$$

By using Table 1, we have

$$
\xi(G)=2 \sum_{p=k} \sum_{i=0} 2^{i} p+4 \sum_{i=1}^{k-1} \sum_{p=k}^{2 k-2} 2^{i} p+2 \sum_{i=k} \sum_{p=k}(2 p-1) 2^{i}
$$

After an easy calculation, we get 


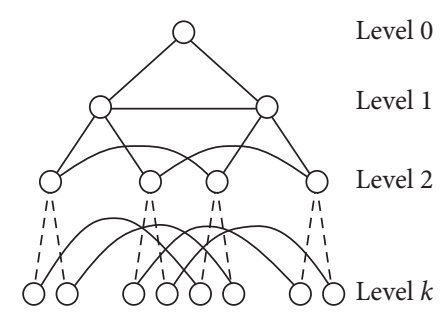

FIGURE 2: $k$-level hypertree HT $(k)$.

TABLE 1: Vertices partition of a hypertree ( $k$-level) based on degree and eccentricity of each vertex with the existence of its frequencies.

\begin{tabular}{lcccc}
\hline$d(v)$ & $\varepsilon(v)$ & Frequency & Range of $i$ & Range of $p$ \\
\hline 2 & $p$ & $2^{i}$ & $i=0$ & $p=k$ \\
4 & $p$ & $2^{i}$ & $1 \leq i \leq k-1$ & $k \leq p \leq 2 k-2$ \\
2 & $2 p-1$ & $2^{i}$ & $i=k$ & $p=k$ \\
\hline
\end{tabular}

$$
\xi(G)=2 k+4 \sum_{i=1}^{k-1} \sum_{p=k}^{2 k-2} 2^{i} p+2^{k+1}(2 k-1) .
$$

The total eccentricity index of a binary tree is computed in the following theorem.

Theorem 2. Consider the graph $G \cong H T(k)$, then the total eccentricity index is equal to

$$
\zeta(G)=k+\sum_{i=1}^{k-1} \sum_{p=k}^{2 k-2} 2^{i} p+(2 k-1) 2^{k}
$$

Proof. Let $G$ be a graph of a hypertree ( $k$-level). To prove (10), we use the total eccentricity index formula:

$$
\zeta(G)=\sum_{u \varepsilon V(G)} \varepsilon(u)
$$

By using Table 1, we get

$$
\zeta(G)=\sum_{p=k} \sum_{i=0} 2^{i} p+\sum_{i=1}^{k-1} \sum_{p=k}^{2 k-2} p \times 2^{i}+\sum_{i=k} \sum_{p=k} 2^{i}(2 p-1) .
$$

After an easy calculation, we get

$$
\zeta(G)=k+\sum_{i=1}^{k-1} \sum_{p=k}^{2 k-2} 2^{i} p+(2 k-1) 2^{k}
$$

The first Zagreb eccentricity index of a binary tree is computed in the following theorem.

Theorem 3. Consider the graph $G \cong H T(k)$, then the first Zagreb eccentricity index is equal to

$$
M_{1}^{\star}(G)=6 k+\sum_{i=1}^{k-1} \sum_{p=k}^{2 k-2}(2 p+1) 2^{i+1}+\sum_{i=1}^{k-1} \sum_{p=k}^{2 k-2}(2 p+2) 2^{i} .
$$

Proof. In order to proof (14), we use the first Zagreb eccentricity index formula:

$$
M_{1}^{\star}(G)=\sum_{u v \in E(G)}\{\varepsilon(u)+\varepsilon(v)\} .
$$

By using Table 2, we get

$$
\begin{aligned}
M_{1}^{\star}(G)= & 3 \sum_{p=k} \sum_{i=0} 2 p 2^{i}+\sum_{i=1}^{k-1} \sum_{p=k}^{2 k-2}(2 p+1) 2^{i+1} \\
& +\sum_{i=1}^{k-1} \sum_{p=k}^{2 k-2}(2 p+2) 2^{i} .
\end{aligned}
$$

After an easy calculation, we get

$$
M_{1}^{\star}(G)=6 k+\sum_{i=1}^{k-1} \sum_{p=k}^{2 k-2}(2 p+1) 2^{i+1}+\sum_{i=1}^{k-1} \sum_{p=k}^{2 k-2}(2 p+2) 2^{i} .
$$

Theorem 4. Consider the graph $G \cong H T(k)$, then the second Zagreb eccentricity index is equal to

$$
M_{1}^{\star \star}(G)=k^{2}+\sum_{i=1}^{k-1} \sum_{p=k}^{2 k-2} p^{2} 2^{i}+(2 k-1)^{2} 2^{k}
$$

Proof. Let $G$ be a graph of a hypertree ( $k$-level). The formula of the second Zagreb eccentricity index is given by

$$
M_{1}^{\star \star}(G)=\sum_{v \in V(G)}[\varepsilon(v)]^{2} .
$$

By using Table 1, we get

$$
M_{1}^{\star \star}(G)=\sum_{p=k} \sum_{i=0} p^{2} 2^{i}+\sum_{i=1}^{k-1} \sum_{p=k}^{2 k-2} p^{2} 2^{i}+\sum_{i=k} \sum_{p=k}(2 p-1)^{2} 2^{i}
$$

After an easy calculation, we get

$$
M_{1}^{\star \star}(G)=k^{2}+\sum_{i=1}^{k-1} \sum_{p=k}^{2 k-2} p^{2} 2^{i}+(2 k-1)^{2} 2^{k}
$$

Theorem 5. Consider the graph $G \cong H T(k)$, then the third Zagreb eccentricity index is equal to

$$
M_{2}^{\star}(G)=3 k^{2}+\sum_{i=1}^{k-1} \sum_{p=k}^{2 k-2} p(p+1) 2^{i+1}+\sum_{i=k} \sum_{p=k}(p+1)^{2} 2^{i} .
$$

Proof. In order to prove (22), we use the formula of the third Zagreb eccentricity index:

$$
M_{2}^{\star}(G)=\sum_{u v \in E(G)} \varepsilon(u) \times \varepsilon(v) .
$$

By using Table 2, we get 
TABLE 2: Edge partition of a hypertree ( $k$-level) based on eccentricity of end vertices of each edge with existence of its frequencies.

\begin{tabular}{lccc}
\hline$(\varepsilon(u), \varepsilon(v))$ & Frequency & Range of $i$ & Range of $p$ \\
\hline$(p, p)$ & $3.2^{i}$ & $i=0$ & $p=k$ \\
$(p, p+1)$ & $2^{i+1}$ & $1 \leq i \leq k-1$ & $k \leq p \leq 2 k-2$ \\
$(p+1, p+1)$ & $2^{i}$ & $1 \leq i \leq k-1$ & $k \leq p \leq 2 k-2$ \\
\hline
\end{tabular}

$$
\begin{aligned}
M_{2}^{\star}(G)= & 3 \sum_{p=k} \sum_{i=0} p^{2} 2^{i}+\sum_{i=1}^{k-1} \sum_{p=k}^{2 k-2} p(p+1) 2^{i+1} \\
& +\sum_{i=k} \sum_{p=k}(p+1)^{2} 2^{i} .
\end{aligned}
$$

After an easy calculation, we get

$$
M_{2}^{\star}(G)=3 k^{2}+\sum_{i=1}^{k-1} \sum_{p=k}^{2 k-2} p(p+1) 2^{i+1}+\sum_{i=1}^{k-1} \sum_{p=k}^{2 k-2}(p+1)^{2} 2^{i} .
$$

The 1-rooted sibling tree $\mathrm{ST}_{k}^{1}$ shown in Figure 3 is obtained from the 1-rooted complete binary tree $T_{k}^{1}$ by adding edges (sibling edges) between the left and right children of the same parent node [16].

An $X$-tree XT $(k)$ shown in Figure 4 is obtained from a complete binary tree on $2^{k+1}-1$ vertices of height $2^{i}-1$ and adding paths $P_{i}$ left to right through all the vertices at level $i$, $1 \leq i \leq k$.

A graph $G_{1}=\left(V_{1}, E_{1}\right)$ is said to be isomorphic to the graph $G_{2}=\left(V_{2}, E_{2}\right)$, if there is a one-to-one correspondence between the vertex sets $V_{1}$ and $V_{2}$ and a one-to-one correspondence between the edge sets $E_{1}$ and $E_{2}$ in such a way that if $e_{1}$ is an edge with end vertices $u_{1}$ and $v_{1}$ in $G_{1}$, then the corresponding edge $e_{2}$ in $G_{2}$ has its end points in the vertices $u_{2}$ and $v_{2}$ in $G_{2}$ which correspond to $u_{1}$ and $v_{1}$, respectively. Such a pair of correspondences is called the graph isomorphism.

Remark 1. $\mathrm{HT}(k) \nRightarrow \mathrm{ST}_{k}^{1}$, but their topological eccentricitybased indices are equal:

$$
\begin{aligned}
\xi(\mathrm{HT}(k)) & =\xi\left(\mathrm{ST}_{k}^{1}\right), \zeta(\mathrm{HT}(k))=\zeta\left(\mathrm{ST}_{k}^{1}\right), M_{1}^{\star}(\mathrm{HT}(k)) \\
& =M_{1}^{\star}\left(\mathrm{ST}_{k}^{1}\right), \\
M_{1}^{\star \star}(\mathrm{HT}(k)) & =M_{1}^{\star \star}\left(\mathrm{ST}_{k}^{1}\right), M_{2}^{\star}(\mathrm{HT}(k))=M_{2}^{\star}\left(\mathrm{ST}_{k}^{1}\right) .
\end{aligned}
$$

Theorem 6. Consider the graph $G \cong X T(k)$ and $k \geq 3$, then the eccentric connectivity index is equal to

$$
\begin{aligned}
\xi(G)= & 2 k+8 \sum_{p=k}^{2 k-2} p+4(2 k-1)+5 \sum_{p=k}^{2 k-3} \sum_{i=1}^{k-2} 2^{i} p+3(2 k-2) 2^{k-1} \\
& 5 \sum_{p=k+2, k>3}^{2 k-2} \sum_{i=1, k>3}^{k-3}\left(2\left(2^{i}-1\right) p\right)+3(2 k-1)\left(2\left(2^{k-2}-1\right)\right) .
\end{aligned}
$$

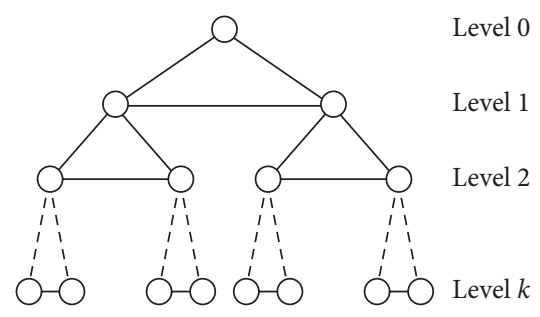

FIGURE 3: 1-rooted sibling tree $\mathrm{ST}_{k}^{1}$.

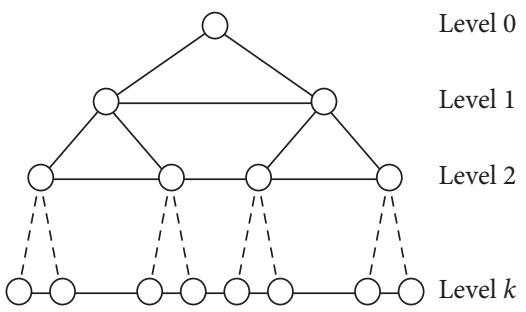

Figure 4: $k$-level $X$-tree XT $(k)$.

Proof. In order to prove the above result, we use the formula of the eccentric connectivity index:

$$
\xi(G)=\sum_{v \in V(G)} d(v) \mathcal{E}(v) .
$$

By using Table 3, we have

$$
\begin{aligned}
\xi(G)= & 2 \sum_{p=k, k \geq 3} p \sum_{i=0} 2^{i}+4 \sum_{i=1} 2^{i} \sum_{p=k, k \geq 3}^{2 k-2} p \\
& +2 \sum_{i=1} 2^{i} \sum_{p=k, k \geq 3}(2 p-1)+5 \sum_{p=k, k \geq 3}^{2 k-3} p \sum_{i=1, k \geq 3}^{k-2} 2^{i} \\
& +5 \sum_{p=k+2, k>3}^{2 k-2} p \sum_{i=1, k>3}^{k-3} 2\left(2^{i}-1\right) \\
& +3 \sum_{p=k, k \geq 3}(2 p-2) \sum_{i=k-1, k \geq 3} 2^{i} .
\end{aligned}
$$

After an easy calculation, we get

$$
\begin{gathered}
\xi(G)=2 k+8 \sum_{p=k}^{2 k-2} p+4 \sum_{p=k}(2 p-1)+5 \sum_{p=k}^{2 k-3} \sum_{i=1}^{k-2} 2^{i} p+3(2 k-2) 2^{k-1} \\
5 \sum_{p=k+2, k>3}^{2 k-2} \sum_{i=1, k>3}^{k-3}\left(2\left(2^{i}-1\right) p\right)+3(2 k-1)\left(2\left(2^{k-2}-1\right)\right) .
\end{gathered}
$$

Theorem 7. Consider the graph $G \cong X T(k)$ and $k \geq 3$, then the total eccentricity index is equal to

$$
\begin{aligned}
\zeta(G)= & k+2 \sum_{p=k}^{2 k-2} p+2(2 k-1)+\sum_{p=k}^{2 k-3} \sum_{i=1}^{k-2} 2^{i} p \\
& +\sum_{p=k+2, k>3}^{2 k-2} \sum_{i=1, k>3}^{k-3}\left(2\left(2^{i}-1\right) p\right) \\
& +(2 k-1)\left(2\left(2^{k-2}-1\right)\right)+(2 k-2) 2^{k-1} .
\end{aligned}
$$


TABle 3: Vertices partition of an $X$-tree ( $k$-level) based on degree and eccentricity of each vertex with the existence of its frequencies.

\begin{tabular}{lcccc}
\hline$d(v)$ & $\varepsilon(v)$ & Frequency & Range of $i$ & Range of $p$ \\
\hline 2 & $p$ & $2^{i}$ & $i=0$ & $p=k$ \\
4 & $p$ & $2^{i}$ & $i=1$ & $k \leq p \leq 2 k-2$ \\
2 & $2 p-1$ & $2^{i}$ & $i=1$ & $p=k$ \\
5 & $p$ & $2^{i}$ & $1 \geq i \geq k-2$ & $k \geq p \geq 2 k-3$ \\
5 & $p$ & $2\left(2^{i}-1\right)$ & $1 \geq i \geq k-3$, & $k+2 \geq p \geq 2 k-2$, \\
3 & $2 p-1$ & $2\left(2^{i}-1\right)$ & $i=k-2$ & $k>3$ \\
3 & $2 p-2$ & $2^{i}$ & $i=k-1$ & $p=k$ \\
\hline
\end{tabular}

Proof. Let $G$ be a graph of an $X$-tree ( $k$-level). In order to prove, we use the total eccentricity index formula:

$$
\zeta(G)=\sum_{u \in V(G)} \varepsilon(u)
$$

By using Table 3, we get

$$
\begin{aligned}
\zeta(G)= & \sum_{p=k} p \sum_{i=0} 2^{i}+\sum_{i=1} 2^{i} \sum_{p=k}^{2 k-2} p+\sum_{i=1} 2^{i} \sum_{p=k}(2 p-1) \\
& +\sum_{p=k}^{2 k-3} p \sum_{i=1}^{k-2} 2^{i}+\sum_{p=k+2, k>3}^{2 k-2} p \sum_{i=1, k>3}^{k-3}\left(2\left(2^{i}-1\right)\right) \\
& +\sum_{p=k}(2 p-1) \sum_{i=k-2}\left(2\left(2^{i}-1\right)\right) \\
& +\sum_{p=k}(2 p-2) \sum_{i=k-1} 2^{i} .
\end{aligned}
$$

After an easy calculation, we get

$$
\begin{aligned}
\zeta(G)= & k+2 \sum_{p=k}^{2 k-2} p+2(2 k-1)+\sum_{p=k}^{2 k-3} \sum_{i=1}^{k-2} 2^{i} p \\
& +\sum_{p=k+2, k>3}^{2 k-2} \sum_{i=1, k>3}^{k-3}\left(2\left(2^{i}-1\right) p\right) \\
& +(2 k-1)\left(2\left(2^{k-2}-1\right)\right)+(2 k-2) 2^{k-1}
\end{aligned}
$$

Theorem 8. Consider the graph $G \cong X T(k)$ and $k \geq 3$, then the first Zagreb eccentricity index is equal to

$$
\begin{aligned}
M_{1}^{\star}(G)= & 12 k+\sum_{i=1}^{k-2} \sum_{p=k+1}^{2 k-2}(2 p)\left(3\left(2^{i}-1\right)\right) \\
& +(4 k-3)\left(2^{k-1}+2\right) \\
& +\sum_{i=1}^{k-2} \sum_{p=k}^{2 k-3}(2 p+1)\left(2\left(3\left(2^{i-1}\right)+1\right)\right) \\
& +(4 k-2)\left(2\left(2^{k-2}-1\right)\right) .
\end{aligned}
$$

Proof. In order to prove the above result, we use the first Zagreb eccentricity index formula:

$$
M_{1}^{\star}(G)=\sum_{u v \in E(G)}\{\varepsilon(u)+\varepsilon(v)\} .
$$

By using Table 4 , we get

$$
\begin{aligned}
M_{1}^{\star}(G)= & \sum_{p=k}(p+p) \sum_{i=0} 6\left(2^{i}\right)+\sum_{i=1}^{k-2} 3\left(2^{i}-1\right) \sum_{p=k+1}^{2 k-2}(p+p) \\
& +\sum_{i=1}^{k-2}\left(2\left(3\left(2^{i-1}\right)+1\right)\right) \sum_{p=k}^{2 k-3}(p+p+1) \\
& +\sum_{p=k}(2 p-2+2 p-1) \sum_{i=k-2}\left(2^{i+1}+2\right) \\
& +\sum_{p=k}(2 p-1+2 p-1) \sum_{i=k-2}\left(2\left(2^{i}-1\right)\right) .
\end{aligned}
$$

After an easy calculation, we get

$$
\begin{aligned}
M_{1}^{\star}(G)= & 12 k+\sum_{i=1}^{k-2} \sum_{p=k+1}^{2 k-2}(2 p)\left(3\left(2^{i}-1\right)\right) \\
& +(4 k-3)\left(2^{k-1}+2\right) \\
& +\sum_{i=1}^{k-2} \sum_{p=k}^{2 k-3}(2 p+1)\left(2\left(3\left(2^{i-1}\right)+1\right)\right. \\
& \left.+(4 k-2)\left(2\left(2^{k-2}-1\right)\right)\right) .
\end{aligned}
$$

Theorem 9. Consider the graph $G \cong X T(k)$ and $k \geq 3$, then the second Zagreb eccentricity index is equal to

$$
\begin{aligned}
M_{1}^{\star \star}(G)= & k^{2}+2 \sum_{p=k}^{2 k-2} p^{2}+2(2 k-1)^{2}+\sum_{p=k}^{2 k-3} \sum_{i=1}^{k-2} 2^{i} p^{2} \\
& +\sum_{p=k+2, k>3}^{2 k-2} \sum_{i=1}^{k-3}\left(2\left(2^{i}-1\right) p^{2}\right) \\
& +(2 k-1)^{2}\left(2\left(2^{k-2}-1\right)\right)+(2 k-2)^{2} 2^{k-1} .
\end{aligned}
$$

Proof. Let $G$ be a graph of an $X$-tree ( $k$-level) and $k \geq 3$. The formula of the second Zagreb eccentricity index is given by

$$
M_{1}^{\star \star}(G)=\sum_{v \in V(G)}[\varepsilon(v)]^{2} .
$$

By using Table 3, we get the following expression:

$$
\begin{aligned}
M_{1}^{\star \star}(G)= & \sum_{p=k} p^{2} \sum_{i=0} 2^{i}+\sum_{i=1} 2^{i} \sum_{p=k}^{2 k-2} p^{2}+\sum_{i=1} 2^{i} \sum_{p=k}(2 p-1)^{2} \\
& +\sum_{i=1}^{k-2} 2^{i} \sum_{p=k}^{2 k-3} p^{2}+\sum_{i=1, k>3}^{k-3}\left(2\left(2^{i}-1\right)\right) \sum_{p=k+2, k>3}^{2 k-2} p^{2} \\
& +\sum_{i=k-2}\left(2\left(2^{i}-1\right)\right) \sum_{p=k}(2 p-1)^{2} \\
& +\sum_{i=k-1} 2^{i} \sum_{p=k}(2 p-2)^{2} .
\end{aligned}
$$


TABLE 4: Edge partition of an $X$-tree ( $k$-level) based on eccentricity of end vertices of each edge with existence of its frequencies.

\begin{tabular}{lccc}
\hline$(\varepsilon(u), \varepsilon(v))$ & Frequency & Range of $i$ & Range of $p$ \\
\hline$(p, p)$ & $6\left(2^{i}\right)$ & $i=0$ & $p=k$ \\
$(p, p)$ & $3\left(2^{i}-1\right)$ & $1 \leq i \leq k-2$ & $k+1 \leq p \leq 2 k-2$ \\
$(p, p+1)$ & $2\left(3\left(2^{i-1}\right)+1\right)$ & $1 \leq i \leq k-2$ & $k \leq p \leq 2 k-3$ \\
$(2 p-2,2 p-1)$ & $2^{i+1}+2$ & $i=k-2$ & $p=k$ \\
$(2 p-1,2 p-1)$ & $2\left(2^{i}-1\right)$ & $i=k-2$ & $p=k$ \\
\hline
\end{tabular}

After an easy calculation, we get

$$
\begin{aligned}
M_{1}^{\star \star}(G)= & k^{2}+2 \sum_{p=k}^{2 k-2} p^{2}+2(2 k-1)^{2}+\sum_{p=k}^{2 k-3} \sum_{i=1}^{k-2} 2^{i} p^{2} \\
& +\sum_{p=k+2, k>3}^{2 k-2} \sum_{i=1}^{k-3}\left(2\left(2^{i}-1\right) p^{2}\right) \\
& +(2 k-1)^{2}\left(2\left(2^{k-2}-1\right)\right)+(2 k-2)^{2} 2^{k-1}
\end{aligned}
$$

Theorem 10. Consider the graph $G \cong X T(k)$ and $k \geq 3$, then the third Zagreb eccentricity index is equal to

$$
\begin{aligned}
M_{2}^{\star}(G)= & 6 k^{2}+\sum_{p=k+1}^{2 k-2} \sum_{i=1}^{k-2} 3\left(2^{i}-1\right) p^{2} \\
& +\sum_{p=k}^{2 k-3} \sum_{i=1}^{k-2} p(p+1)\left(2\left(3\left(2^{i-1}\right)+1\right)\right) \\
& +(2 k-1)(2 k-2)\left(2^{k-1}+2\right) \\
& +(2 k-1)^{2}\left(2\left(2^{k-2}-1\right)\right) .
\end{aligned}
$$

Proof. In order to prove the above result, we use the formula of the third Zagreb eccentricity index:

$$
M_{2}^{\star}(G)=\sum_{u v \in E(G)} \varepsilon(u) \times \varepsilon(v) .
$$

By using Table 4, we get

$$
\begin{aligned}
M_{2}^{\star}(G)= & \sum_{p=k}(p)^{2} \sum_{i=0} 6\left(2^{i}\right)+\sum_{i=1}^{k-2}\left(3\left(2^{i}-1\right)\right) \sum_{p=k+1}^{2 k-2}(p)^{2} \\
& +\sum_{i=1}^{k-2}\left(2\left(3\left(2^{i-1}\right)+1\right)\right) \sum_{p=k}^{2 k-3} p(p+1) \\
& +\sum_{i=k-2}\left(2^{i+1}+2\right) \sum_{p=k}(2 p-2)(2 p-1) \\
& +\sum_{i=k-2}\left(2\left(2^{i}-1\right)\right) \sum_{p=k}(2 p-1)(2 p-1) .
\end{aligned}
$$

After an easy calculation, we get

$$
\begin{aligned}
M_{2}^{\star}(G)= & 6 k^{2}+\sum_{p=k+1}^{2 k-2} \sum_{i=1}^{k-2} 3\left(2^{i}-1\right) p^{2} \\
& +\sum_{p=k}^{2 k-3} \sum_{i=1}^{k-2} p(p+1)\left(2\left(3\left(2^{i-1}\right)+1\right)\right) \\
& +(2 k-1)(2 k-2)\left(2^{k-1}+2\right) \\
& +(2 k-1)^{2}\left(2\left(2^{k-2}-1\right)\right) .
\end{aligned}
$$

\section{Conclusion}

In this paper, we have computed the eccentricity-based topological indices such as the eccentric connectivity index, the total eccentricity index, and the first, second, and third Zagreb eccentricity index for certain interconnection networks such as a hypertree, sibling tree, and $X$-tree $k$-level. These results are useful in topological characterization of these important chemical networks.

\section{Data Availability}

The data used to support the findings of this study are included within the article.

\section{Conflicts of Interest}

The authors declare that there are no conflicts of interest regarding the publication of this paper.

\section{Acknowledgments}

This work was supported by the research foundation of Huainan Normal University (Grant no. 2019XJZD05) and UPAR grants of United Arab Emirates University (Grant nos. G00002590 and G00003271).

\section{References}

[1] S. Hayat and M. Imran, "Computation of topological indices of certain networks," Applied Mathematics and Computation, vol. 240, pp. 213-228, 2014.

[2] M. Imran, A. Q. Baig, and H. Ali, "On topological properties of dominating David derived networks," Canadian Journal of Chemistry, vol. 93, pp. 730-739, 2015.

[3] M. Imran, S. Hayat, and M. Y. H. Malik, "On topological indices of certain interconnection networks," Applied Mathematics and Computation, vol. 244, pp. 936-951, 2014.

[4] T. W. Haynes, S. M. Hedetniemi, S. T. Hedetniemi, and M. A. Henning, "Power domination in graphs applied to electrical power networks," SIAM Journal on Discrete Mathematics, vol. 15, no. 4, pp. 519-529, 2002.

[5] R. S. Rajana, J. Anithaa, and I. Rajasingha, "2-power domination in certain interconnection networks," Procedia Computer Science, vol. 57, pp. 738-744, 2015.

[6] V. Sharma, R. Goswami, and A. K. Madan, "Eccentric connectivity index: a novel highly discriminating topological descriptor for structure property and structure activity studies," Journal of Chemical Information and Computer Sciences, vol. 37, pp. 273-282, 1997. 
[7] R. Farooq and M. Ali Malik, "On some eccentricity based topological indices of nanostar dendrimers," Optoelectronics and advanced materials-rapid communications, vol. 9, pp. 842-849, 2015.

[8] I. Gutman and N. Trinajstić, "Graph theory and molecular orbitals total $\varphi$-electron energy of alternant hydrocarbons," Chemical Physics Letters, vol. 17, no. 4, pp. 535-538, 1972.

[9] M. Ghorbani and M. A. Hosseinzadeh, "A new version of Zagreb indices," Filomat, vol. 26, no. 1, pp. 93-100, 2012.

[10] S. Hayat and M. Imran, "Computation of certain topological indices of nanotubes," Journal of Computational and Theoretical Nanoscience, vol. 12, no. 1, pp. 70-76, 2015.

[11] S. Hayat, M. A. Malik, and M. Imran, "Computing topological indices of honey-comb derived networks," Romjist, vol. 18, pp. 144-165, 2015.

[12] S. Hayat and M. Imran, "Computation of certain topological indices of nanotubes covered by $C_{5}$ and $C_{7}$," Journal of Computational and Theoretical Nanoscience, vol. 12, pp. 533-541, 2015.

[13] S. Hayat and M. Imran, "On degree based topological indices of certain nanotubes," Journal of Computational and Theoretical Nanoscience, vol. 12, no. 8, pp. 1599-1605, 2015.

[14] F. F. Dragan and A. Brandstadt, " $r$-dominating cliques in graphs with hypertree structure," Discrete Mathematics, vol. 162, pp. 93-108, 1996.

[15] M. Baca, J. Horvathova, M. Mokrisova, and A. Suhanyiova, "On topological indices of fullerenes," Applied Mathematics and Computation, vol. 251, pp. 154-161, 2015.

[16] R. S. Rajan, I. Rajasingh, T. M. Rajalaxmi, and N. Parthiban, "Embedding of circulant networks into $k$-rooted sibiling trees," International Journal of Pure and Applied Mathematics, vol. 86, no. 6, pp. 1005-1012, 2013. 